\title{
ANÁLISE DOS ELEMENTOS CLIMÁTICOS E AS NOTIFICAÇÕES DE DENGUE EM SÃO LUÍS, MARANHÃO, BRASIL
}

\author{
Marco Aurélio Neri Torres ${ }^{(a)}$, Audivan Ribeiro Garcês Júnior ${ }^{(b)}$, Priscilla Coelho Ribeiro ${ }^{(c)}$, José \\ Aquino Junior ${ }^{(\mathrm{d})}$ \\ (a) Departamento de Geociências, Universidade Federal do Maranhão, marco.torres.geo@gmail.com \\ (b) Mestre em Saúde e Ambiente - PPGSA, Universidade Federal do Maranhão, audivanribeiro@ gmail.com \\ (c) Departamento de Oceanografia e Liminologia, Universidade Federal do Maranhão, pris.coelho.slz@gmail.com \\ (d) Professor Adjunto - Departamento de Geociências, Universidade Federal do Maranhão, zeaquinoju@ gmail.com
}

\section{Eixo: Climatologia em diferentes níveis escalares: mudanças e variabilidades}

\begin{abstract}
Resumo
Este estudo tem como objetivo identificar as possíveis correlações entre as variáveis climáticas de São Luís com os casos notificados de dengue, fazendo isso por meio de Regressão e PCA no período de 2012 a 2015. Os dados climáticos foram disponibilizados pelo INMET, analisou-se as variáveis de temperatura (máxima, média e mínima), umidade relativa, pressão atmosférica, precipitação e os dados de casos de dengue, disponibilizados pela Secretaria Estadual de Sáude do Maranhão. Estes dados foram analisados utilizando o software Statistica 7.0. Foi obtido como resultado que a correlação entre a umidade e pluviosidade e os casos de dengue. A temperatura apresentou correlação forte, porem negativa; a pressão atmosférica apresentou correlação fraca com a dengue.
\end{abstract}

Palavras chave: Climatologia; São Luís; dengue; PCA

\section{Introdução}

O clima influi diretamente na vida da sociedade, uma vez que estabelece uma relação direta com o homem e seu meio de convívio social (cidades, áreas rurais, regiões, etc.). Pela dinâmica socioambiental existentes nas cidades e a junção de fatores adversos vindos da urbanização, o estudo do clima nessas áreas encontrase como uma vertente crescente dentro da climatologia brasileira, estudos fortemente influenciados por metodologias como a do Sistema Clima Urbano (S.C.U) proposto por Monteiro (1976).

A dengue é uma doença encontrada principalmente em países de clima tropical, onde os fatores climáticos como temperatura e umidade são perfeitos para a proliferação do mosquito Aedes aegypti, transmissor do vírus. Conforme afirma Mendonça, Souza e Dutra (2009, p. 264), “A situação atual e preocupante da dengue no Brasil reflete um complexo contexto no qual se interagem ineficácias gerais de atuação do poder público e da sociedade em geral."

A cidade de São Luís, capital do Estado do Maranhão, segundo o IBGE (2010), possui uma população de 1.014.837 habitantes, estando assim dentro do grupo de cidades que são identificadas como grandes metrópoles de caráter urbano no Brasil. Além desses fatores, a grande aglomeração urbana de São Luís, 
XVII Simpósio Brasileiro

de Geografia Fisica Aplicada

I Congresso Nacional

de Geografia Física
OS DESAFIOS DA GEOGRAFIA FÍSICA NA FRONTEIRA DO CONHECIMENTO

Instituto de Geociências - Unicamp

Campinas - SP

28 de Junho à 02 de Julho de 2017

constituída pelos municípios de São José de Ribamar, Raposa e Paço do Lumiar, formam uma área contínua de grande urbanização provenientes das últimas duas décadas.

Em São Luís, observando-se que a incidência dos casos de dengue vem aumentando consideravelmente, percebendo-se um aumento expressivo nos últimos anos, e os fatores que envolvem a variabilidade climática de uma cidade podem estar diretamente ligados ao aumento expressivo no vetor da dengue no município.

Para a efetivação dos dados utilizou-se técnicas estatísticas, que para Sartori (2008), já demonstrava preocupação em relação a estatística mais adequada a estudos que envolvam fatores ambientais e biológicas observando que "os métodos multivariados são modelos estatísticos que consideram muitas variáveis ao mesmo tempo, o que demanda um exame detalhado e rigoroso dos dados, pois o tratamento inadequado pode ter efeitos catastróficos".

O presente estudo tem por objetivo identificar as possíveis correlações entre as variáveis climáticas com os casos notificados de dengue em São Luís, por meio de Regressão linear múltipla e PCA (Principal Component Analysis), para os anos de 2012 a 2015.

\section{Metodologia}

Primeiramente foi realizada a busca por dados das variáveis climáticas a citar: a temperatura (máxima, média, mínima), precipitação, pressão atmosférica e umidade relativa do ar. Optou-se pela utilização de dados da estação meteorológicas situada em São Luís-MA, localizada entre a latitude - 02.53 e longitude 44.21, onde a mesma pertence ao Instituto Nacional de Meteorologia (INMET), cujos dados são disponibilizados por meio do site <http://www.inmet.gov.br/portal/>. Sendo assim, os dados foram coletados na escala diária e depois, para possibilitar as análises estatísticas, organizados em médias mensais para as variáveis de temperatura, umidade e pressão atmosférica. Já os dados de precipitação foram organizados através da soma dos seus valores absolutos.

Quando foi feita a tabulação dos dados disponibilizados pela estação meteorológica do INMET, percebeuse que em algumas medições havia perda de dados por algum problema nos sensores, sendo assim, no período de 13/10/2014 a 12/11/2013 não foram disponibilizados os dados de temperatura máxima ar e temperatura média do ar e de 13/11/2013 a 31/12/2013 para pressão atmosférica; ja no ano de 2014, não foram disponibilizados dados de Pressão Atmosférica entre o período de 12/10/2014 a 13/11/2014. Quanto aos casos notificados de dengue, foram utilizados dados da Secretaria Estadual de Saúde do Maranhão, os 
quais são banco de dados primários de agravos em saúde, sendo coletados em escala diária e posteriormente agrupados para a escala mensal de análise.

Os dados foram organizados em planilhas do microsoft Excel 2013 para a realização dos testes estatísticos, afim de que se pudesse perceber o comportamento desses dados. Os testes foram feitos através do software STATISTICA 7.0 free version.

No presente trabalho utilizou uma Regressão linear para verificar a relação entre as variáveis climáticas sobre a variável dengue, por meio do coeficiente de determinação $\mathrm{R}^{2}$ e ANOVA para verificar diferença entre as amostras testadas, verificando $p$-valor significativo quando $\leq 0,05$. Para avaliar a relação visualizada teste anterior, foi aplicado uma análise de componente principal (PCA "Principal Component Analysis”) de base na correlação dos itens, diminuindo a discrepância dos possíveis outlier.

A regressão linear múltipla é uma técnica cuja finalidade é descrever a relação matemática de uma variável dependente (variável estudada) com as variáveis independentes (variáveis que tentam explicar a variável de estudo), sendo assim, explicando o efeito de cada variável independente sobre a estudada. Esta técnica permite a detecção dos principais padrões de similaridade, associação e correlação entre as variáveis.

Sua fórmula é dada por:

$y=\beta 0+\beta i x i+\ldots+\beta n x n+\varepsilon$

onde : y é a variável resposta;

$\mathbf{x i}(i=1,2, \ldots, \mathbf{n})$ são as variáveis explicativas.

及0 representa o valor de $\mathbf{y}$ quando as variáveis explicativas são nulas,

Bi são chamados de coeficientes de regressão

$\boldsymbol{\varepsilon}$ (resíduo) é a diferença entre os valores reais e os previstos da variável resposta, que é assumido normalmente distribuído com média zero e variância $\boldsymbol{\sigma 2}$ (HAIR Jr. et al., 2005).

A análise de componente principal é uma análise estatística que permite avaliar um conjunto de variáveis a partir das respectivas matrizes de variância-covariância ou correlação, com a perda mínima de dados. A partir desse método, as variáveis originais geram novas variáveis, as componentes principais. A primeira componente $(\mathrm{CP} 1)$ é a responsável pela \% mais alta de explicação dos dados originais, quando considerado o valor das matrizes, sendo aparecem em ordem decrescente de \% das variáveis originais $(\mathrm{CP} 2, \mathrm{CP} 3, \ldots \mathrm{CPn})$, sendo a soma das componente $100 \%$ da explicação, sendo que quanto maior for a correlação entre as medidas originais, maior será a parcela de variação explicada pelos primeiros dois 


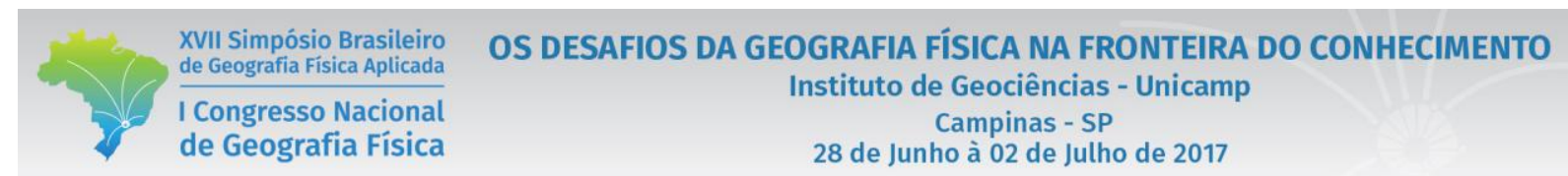

eixos (GOMES, 1985; LANDIM, 2000). Ela é indicada para conjunto de dados correlacionado linearmente (MANLY, 1994).

Neste estudo, utilizou-se a matriz de correlação para gerar os valores de porcentagem de variabilidade das variáveis para cada componente. Segundo Bernardi (2001), estes valores, ao serem multiplicados pela matriz principal, forneceram a matriz de "scores" que foram dispostos em um diagrama de dispersão bidimensional com as duas componentes de maior \% de explicação dos dados. Os valores positivos e negativos das variáveis dentro de cada componente indicam relações direta e inversamente proporcionais.

\subsection{Localização e Caracterização Climática área de estudo}

A cidade São Luís (Figura 01), limita-se com o Oceano Atlântico, ao Norte; com o Estreito dos Mosquitos, ao Sul; com a Baía de São Marcos, a Oeste e com o município de São José de Ribamar, a Leste. Segundo (IBGE, 2010) Tem uma área de 827 km², localizada na Latitude: 02³1'47’'S e Longitude: $44^{\circ} 18^{\prime} 10^{\prime \prime} \mathrm{W}$.

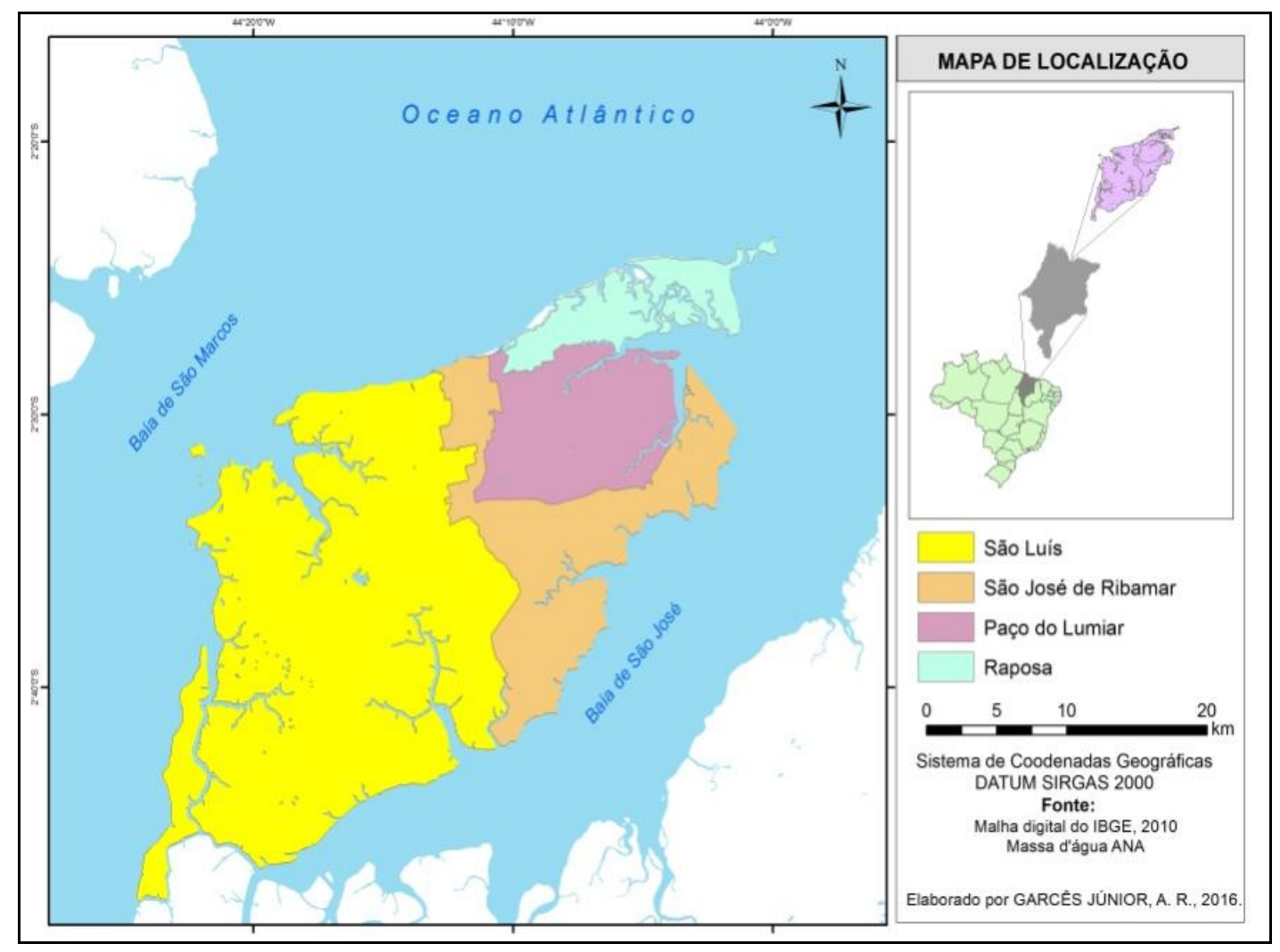

Figura 1. Mapa de localização do município de São Luís.

Fonte: Elaborado a partir de IBGE, 2010. 
O município de São Luís está dentro da área compreendida como zona tropical, recebendo altos índices de radiação solar, que irá refletir nas altas temperaturas médias (FEITOSA \& TROVÃO, 2006). Segundo proposta de Mendonça e Danni-Oliveira (2007), para classificação dos domínicos climáticos do Brasil, o norte Maranhense, onde situa-se São Luís, caracteriza-se por um clima tropical equatorial 2a (com quatro a cinco meses secos). Para Mendonça \& Danni-Oliveira (2007, p. 159) este clima "se caracteriza tanto pela influência da maritimidade quanto da continentalidade. As temperaturas, mesmo elevadas ao longo do ano, apresentam pequena variação sazonal e a pluviosidade, maior que os índices térmicos”.

Em São Luís tem-se duas estações bem definidas, havendo regularidade térmica durante todo ano que são definidas pelo regime pluviométrico, há um período chuvoso, compreendido entre o verão e outono, e outro seco, entre o inverno e a primavera (MENDONÇA \& DANNI-OLIVEIRA, 2007).

Esta definição é possível a partir da compreensão da atuação dos sistemas atmosféricos atuantes sobre a região, estes estão relacionados às mudanças do equador térmico. Segundo Nimer e Brandão (1989 citado por ARAÚJO, 2014, p.94) “ao longo da depressão equatorial, caracterizada por uma região de pressões relativamente baixas e ventos calmos, o ar instável provoca chuvas e trovoadas bastante intensas". As convergências das massas de ar nestas áreas de baixas pressão no equador geram a Zona de Convergência Intertropical (ZCIT) que é determinante para compreensão do regime pluviométrico de todo nordeste brasileiro.

O período de maior influência da ZCIT é a partir do mês de janeiro, com seu deslocamento meridional, estabelecendo um regime de pluviosidade mais regular nos meses de março e abril, com elevados índices de precipitação (FEITOSA, 1996).

Com relação às normais climatológicas, segundo INMET a temperatura média anual fica entre $26,9^{\circ} \mathrm{C}$, sendo os meses de novembro e dezembro os mais quentes e fevereiro, março e julho os mais frios. Com relação à pluviosidade, possui acumulado anual normal de 2.290 mm, sendo os meses de março e abril os mais chuvosos, com $428 \mathrm{~mm}$ e $476 \mathrm{~mm}$, respectivamente, enquanto o mês mais secos é outubro com 7,6 $\mathrm{mm}$. Os ventos predominantes são os de nordeste e apresenta umidade relativa elevada durante todo o ano (INMET, 2009).

\section{Resultados e Discussão}

No total foram 5.761 casos notificados de Dengue no município de São Luís para o período analisado, sendo o ano de 2015 com maior incidência, quantificando 2.408 casos, seguido de 2012 (1.265), 2013 (1.120) e 2014 (968). 
OS DESAFIOS DA GEOGRAFIA FÍSICA NA FRONTEIRA DO CONHECIMENTO

Instituto de Geociências - Unicamp

Campinas - SP

28 de Junho à 02 de Julho de 2017

As variáveis climáticas analisadas apresentaram pouca variação. As variáveis de temperatura não apresentaram grande amplitude térmica nos valores médio observados para os anos estudados, apresentando flutuação de $1,41^{\circ} \mathrm{C}$ para temperatura máxima, tendo sua média anual menor de $30,99^{\circ} \mathrm{C}$ em 2013 , e a maior de $32,40^{\circ} \mathrm{C}$ em 2015 ; de $0,54^{\circ} \mathrm{C}$ para temperatura média, possuindo o menor valor anual entre os anos de $26,84^{\circ} \mathrm{C}$ em 2014 e o maior $27,38^{\circ} \mathrm{C}$ em 2012 ; e $0,31^{\circ} \mathrm{C}$ para temperatura mínima anual, possuindo mínimo de $24,61^{\circ} \mathrm{C}$ em 2014 , e o máximo de $24,92^{\circ} \mathrm{C} \mathrm{em} 2013$. A umidade relativa apresentou valor máximo de $81,15 \%$ (2014) e mínimo de 79,27\% (2015). A pressão apresentou valores médios anuais muito semelhantes, entorno de 1004,96 mbar. A precipitação, analisada para os valores absolutos anuais de precipitação total, apresentou variações significativas com o máximo registado de 1833,90 mm (2014) e mínimo de 1133,20 mm (2012), conforme pode ser observado na Tabela I.

Tabela I: Acumulados anuais das notificações em dengue e as variáveis climáticas de 2012 a 2015.

\begin{tabular}{|c|c|c|c|c|c|c|c|}
\hline Anos & dengue & precipitação & $\begin{array}{c}\text { pressão } \\
\text { atmosférica }\end{array}$ & $\begin{array}{c}\text { temperatura } \\
\text { máxima }\end{array}$ & $\begin{array}{c}\text { temperatura } \\
\text { média }\end{array}$ & $\begin{array}{c}\text { temperatura } \\
\text { mínima }\end{array}$ & $\begin{array}{c}\text { umidade } \\
\text { relativa }\end{array}$ \\
\hline 2012 & 1265 & 1133.2 & 1005.30 & 32.24 & 27.38 & 24.66 & 80.80 \\
\hline 2013 & 1120 & 1594.2 & 1002.87 & 30.99 & 27.12 & 24.92 & 80.44 \\
\hline 2014 & 968 & 1833.9 & 1005.91 & 31.62 & 26.84 & 24.61 & 81.15 \\
\hline 2015 & 2408 & 1507.2 & 1005.77 & 32.40 & 27.06 & 24.78 & 79.27 \\
\hline Max & & 1833.90 & 1005.91 & 32.40 & 27.38 & 24.92 & 81.15 \\
\hline Min & & 1133.20 & 1002.87 & 30.99 & 26.84 & 24.61 & 79.27 \\
\hline
\end{tabular}

Fonte: Elaborado a partir de dados do INMET.

A aplicação do modelo de Regressão Linear Múltipla mostrou $R^{2}$ superior a 0,76 para as variáveis independentes (temperatura, umidade, pressão e precipitação) para com a variável dengue, sendo estas diferenças estatisticamente significativa apenas para os anos de 2012 e 2015 com p-valor de 0,019 e 0,021 , respectivamente.

Os anos de 2013 e 2014, embora tenha um coeficiente de determinação forte, suas relações apresentaram p-valor superior a 0,05 , podendo dizer que não há significância estatística das variáveis independentes sobre a dengue para estes anos. Estes resultados de $p$-valor para os anos específicos podem ter ocorrido mediante as diferenças em alguma das variáveis, por exemplo, o número dos casos de dengue foi relativamente baixo em relação ao demais anos, isso pode ocorrer por exemplo devido a soro prevalências de muitas pessoas em relação a um sorotipo especifico da dengue (2013), e a perca de dados climáticos em 2013. 
Tabela II. Valores de Coeficiente de correlação, coeficiente de determinação, coeficiente de determinação ajustado e ANOVA para análise de Regressão Linear Múltipla (Pearson) do período de 2012 a 2015.

\begin{tabular}{|l|c|c|r|r|}
\hline \multirow{2}{*}{ Análise } & \multicolumn{4}{c|}{ Ano } \\
\cline { 2 - 5 } & 2012 & 2013 & \multicolumn{1}{c|}{2014} & \multicolumn{1}{c|}{2015} \\
\hline $\mathrm{R}$ & 0,952 & 0,873 & 0,901 & 0,950 \\
\hline $\mathrm{R}^{2}$ & 0,906 & 0,762 & 0,811 & 0,902 \\
\hline $\mathrm{R}^{2}$ ajustado & 0,792 & 0,477 & 0,433 & 0,784 \\
\hline $\mathrm{F}(6,5)$ & 7,986 & 2,672 & 2,145 & 7,642 \\
\hline Erro padrão & 29,040 & 56,337 & 30,536 & 75,678 \\
\hline $\mathrm{F}$ & 7,986 & 2,672 & 2,145 & 7,642 \\
\hline$p$-valor & 0,019 & 0,150 & 0,284 & 0,021 \\
\hline Grau de liberd. & 6 & 6 & 6 & 6 \\
\hline
\end{tabular}

Fonte: Elaborado a partir do Software STATISTICA 7.0

Para a PCA, as componentes explicaram no mínimo 79,48 \% as variáveis analisadas (2013) e máxima explicativa de 94,58\% (2012), valores esses considerados satisfatórios segundo Kaiser (1960). A Componente Principal 1 (CP1) explicou um mínimo de 51,89\% das variáveis de 2013 e máximo de $71,71 \%$ das de 2015 , sendo assim o principal fator de base das análises aqui apresentadas. A Componente Principal 2 (CP2), foi o eixo complementar das explicações mais prováveis para as variáveis, enquadrando o segundo grupo de informações, sendo a menor explicação desta componente de $17,04 \%$ em 2015 e a maior de 27,59\% para o grupo de variáveis de 2013 (Tabela III).

Tabela III. Valores das principais componentes de explicação da PCA para o período de 2012 a 2015.

\begin{tabular}{|c|c|c|c|}
\hline Anos & $\begin{array}{c}\text { \% de Explicação } \\
\text { da CP1 }\end{array}$ & $\begin{array}{c}\text { \% de Explicação } \\
\text { da CP2 }\end{array}$ & $\begin{array}{c}\text { \% de Explicação } \\
\text { da PCA }\end{array}$ \\
\hline 2012 & 69,4 & 25,18 & 94,58 \\
\hline 2013 & 51,89 & 27,59 & 79,48 \\
\hline 2014 & 70,63 & 17,89 & 88,52 \\
\hline 2015 & 71,71 & 17,04 & 88,75 \\
\hline
\end{tabular}

Fonte: Elaborado a partir do Software STATISTICA 7.0

Considerando que a CP1 foi a componente que melhor explicou a maioria das variáveis, observamos que as variáveis precipitação e umidade relativa tem uma correlação positiva com a dengue, sendo esperado um aumento dos casos de dengue com o aumento dos valores destas variáveis. Já as variáveis relacionadas a temperatura (temperatura máxima, média e mínima) se mostraram inversamente proporcionais a variável dengue (Figura 2). 
A partir dos valores de correlação por PCA para as temperaturas, pode-se explanar que é sempre esperado uma diminuição dos casos a medida que os valores aumentam excessivamente, isso se dá porque no segundo semestre em que observamos as maiores temperaturas também ocorre os menores indicies pluviométricos sendo está variável, associada a diversidade dos ambientes urbanos, a mais significante para o desenvolvimento do vetor da dengue.

Considerando a correlação apresentada na Tabela IV para a CP supracitada, os valores de correlação se apresentaram altos para todas as variáveis relacionadas com a dengue, exceto para pressão atmosférica com valor máximo de correlação fraca positiva de 0,252 e negativa de $-0,303$, sendo estes valores de baixa correlação. Nos anos de 2013 e 2015, a pressão atmosférica apresentou correlação quase nula com a dengue. As temperaturas tiveram correlação alta negativa com a variável dengue, exceto para o ano de 2013 quando a temperatura máxima apresentou correlação fraca negativa de - 0,022 na CP1 isso acaba sendo explicável pela perda de dados que ocorreu no ano entre os meses de outubro e novembro (conforme explicado na metodologia), meses estes considerados de altas temperaturas e de baixas pluviométricas, podendo influenciar nos resultados estudados para o ano. Nos demais anos as correlações variaram de -0,861 a - 0,972 , sendo estas correlações forte. A umidade relativa mostrou correlação forte positiva com a dengue (mín.: 0,935 em 2012; máx.: 0,975 em 2014), assim como a variável precipitação (mín.: 0,779 em 2013; máx.: 0,862 em 2015).

Tabela IV. Valores de explicação de cada variável baseada em matriz de correlação com a variável dengue para os anos de 2012, 2013, 2014 e 2015.

\begin{tabular}{|l|c|c|r|r|r|r|r|r|}
\hline \multirow{2}{*}{ Variáveis } & \multicolumn{2}{|c|}{2012} & \multicolumn{2}{c|}{2013} & \multicolumn{2}{c|}{2014} & \multicolumn{2}{c|}{2015} \\
& \multicolumn{1}{|c|}{ CP1 } & CP2 & \multicolumn{1}{c|}{ CP1 } & CP2 & CP1 & CP2 & CP1 & CP2 \\
\hline Precipitação & 0,800 & $-0,532$ & 0,779 & $-0,447$ & 0,797 & $-0,144$ & 0,862 & 0,154 \\
\hline Pressão atmosférica & 0,252 & 0,959 & $-0,097$ & 0,901 & $-0,303$ & 0,934 & $-0,056$ & 0,996 \\
\hline Temperatura máxima & $-0,948$ & 0,218 & $-0,022$ & 0,629 & $-0,928$ & 0,096 & $-0,954$ & 0,083 \\
\hline Temperatura média & $-0,972$ & $-0,137$ & $-0,885$ & $-0,283$ & $-0,971$ & $-0,176$ & $-0,977$ & 0,012 \\
\hline Temperatura mínima & $-0,861$ & $-0,467$ & $-0,902$ & $-0,381$ & $-0,870$ & $-0,376$ & $-0,863$ & 0,003 \\
\hline Umidade relativa & 0,935 & $-0,154$ & 0,949 & $-0,153$ & 0,975 & $-0,011$ & 0,974 & 0,018 \\
\hline
\end{tabular}

Fonte: Elaborado a partir do Software STATISTICA 7.0 

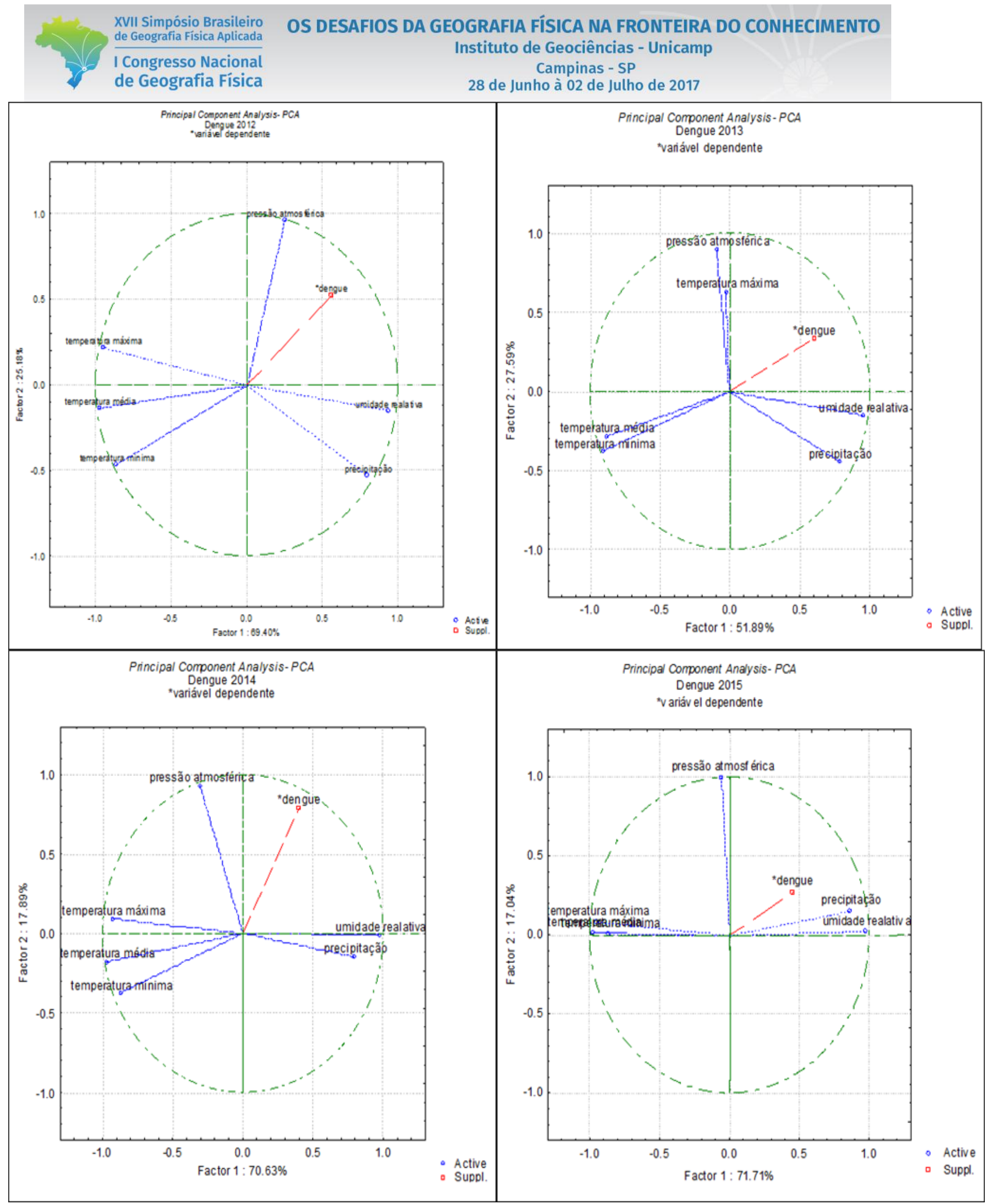

Figura 2. Representação gráfica das variáveis para CP1 (Fator 1) e CP2 (Fator 2) na PCA (Principal Component Analysis) para os anos de 2012, 2013, 2014 e 2015.

Fonte: Elaborado a partir do Software STATISTICA 7.0 


\section{Conclusão}

As análises de Regressão e PCA foram instrumentos de suma importância na evidenciação da relação entre as variáveis estudadas em relação a dengue, proporcionando entender de que forma cada variável se relaciona com a mesma. Para melhor compreensão das influencias das variáveis, recomenda-se um estudo que aplique tais métodos observando a flutuação dos casos em relação aos meses fazendo a utilização de dados diários, uma vez que tais variáveis apresentam grande diferenciação nos valores mensais, sendo possível encontrar estatisticamente resultados mais expressivos.

Os dados aqui apresentados demostram que uma correlação forte das variáveis para os anos de 2012 a 2015, sendo a precipitação e umidade as variáveis que apresentaram um comportamento diretamente proporcional as variações de notificação de caso de dengue. Observou-se um aumento de caso a medida que a precipitação e umidade também aumentam. Já as variáveis de temperatura apresentaram uma correlação forte, mas inversamente proporcional, mostrando uma tendência a diminuição de notificações com o aumento excessivo na temperatura local. A variável pressão atmosfera apresentou correlação muito baixa, sendo esta considerada de pouco influencia para os casos de dengue registrados.

Evidencia-se a importância do clima tropical: temperatura, umidade e regimes pluviométricos sazonais, caracterizado pelo ritmo intermitente de chuvas concentradas, visto que estes possuem dentre os outros elementos meteorológicos analisados, maior relação com o ciclo epidemiológico da doença.

\section{Bibliografia}

ARAÚJO, Ronaldo Rodrigues. Clima e vulnerabilidade socioespacial: uma avaliação dos fatores de risco na população urbana do município de São Luís (MA). Tese. (Doutorado em Geografia). Faculdade de Ciências e Tecnologia, Universidade Estadual Paulista “Júlio de Mesquita Filho", Presidente Prudente, São Paulo, 2014.

BERNARDI, J.V.E.; FOWLER, H.G.; LANDIM, P.M.B. Um estudo de impacto ambiental utilizando análises estatísticas espacial e multivariada. Holos Environmental, 1: 162-172. 2001

FEITOSA, A. C. Dinâmica dos processos geomorfológicos da área costeira a nordeste da Ilha do Maranhão. Rio Claro (SP), 1996. 249f. Tese. (Doutorado em Geografia). Instituto de Geociências e Ciências Exatas, Universidade Estadual Paulista "Júlio de Mesquita Filho", 1996.

FEITOSA, Antonio Cordeiro; TROVÃO, José Ribamar. Atlas escolar do Maranhão: espaço geo-histórico e cultural. João Pessoa: Grafset, 2006.

GOMES, M. B. Determinação de Componentes Principais. Ciência \& Cultura, v. 37, p. 10-12, 1985.

HAIR Jr., J. F. et al. Análise multivariada de dados. São Paulo: Bookman, 2005. 
IBGE, Instituto Brasileiro de Geografia e Estatística. Bases de Informações do Censo Demográfico 2010: Rio de Janeiro: IBGE, 2010.

INMET. Instituto Nacional de Meteorologia. Normais Climatológicas do Brasil 1961 a 1990. Brasília, 2009.

KAISER, H.F. The varimax criteria for analytical rotation in fator analysis. Psychometric, 23, p. $141-151$, 1960.

LANDIM, P.M.B. Análise estatística de dados geológicos multivariados. Lab. Geomatemática, DGA, IGCE,UNESP/Rio Claro, 2000, Texto Didático 03, 128 pp. Disponível em <http://www.rc.unesp.br/igce/aplicada/textodi.html>. Acesso em 22/02/2004.

MANLY, B.F.J. Multivariate statistical methods: a primer. CRC Press, 2004.

MENDONÇA, F.; de. A.; SOUZA, A. V. e.; DUTRA, D. De A. Saúde Pública, Urbanização e Dengue no Brasil. Sociedade \& Natureza, Uberlândia, 21 (3): 257-269, dez. 2009.

MENDONÇA, Francisco e DANNI-OLIVEIRA, Inês M. Climatologia: noções básicas e climas do Brasil. v. 1. São Paulo: Oficina de Texto, 2007.

MONTEIRO, C. A. F. Teoria e Clima Urbano. Série Teses e Monografias, n²5. São Paulo: Instituto de Geografia/USP, 1976.181p. 